\title{
The Image Study of Articular Cartilage in China
}

\author{
Fei Wang $\cdot$ Si Shen $\cdot$ Lin Qiu $\cdot$ Cici Zhang $\cdot$ \\ Meng Chen $\cdot$ Sirun Liu
}

Published online: 12 September 2014

(C) Springer Science+Business Media New York 2014

\begin{abstract}
The purpose of this article is to review the current image study of articular cartilage in China, the conventional and new MRI sequences to be used in cartilage, the clinical application of MRI, Our team's achievements, and future directions and developments in cartilage imaging. In conclusion, articular cartilage plays an important role in the function of the joints of the body. MRI is a noninvasive method for the assessment of cartilage and it can reveal the morphological and biochemical changes in cartilage.
\end{abstract}

Keywords Cartilage - Articular cartilage - MRI ·

Osteoarthritis · Joint · Quantitive MR imaging

\section{Introduction}

Cartilage is one of the most important markers in degenerative and traumatic joint diseases, and the integrity of

This article is part of the Topical Collection on Highlights of Musculoskeletal Imaging in China.

F. Wang $\cdot$ S. Shen $\cdot$ L. Qiu $\cdot$ C. Zhang $\cdot$ M. Chen $\cdot$ S. Liu ( $\square)$ Department of Medical Imaging, The First Affiliated Hospital, Jinan University, 613\#, West of Huang Pu Street, Guangzhou 510630, Guang Dong, People's Republic of China e-mail: tlsr@jnu.edu.cn

F. Wang

e-mail: 412470138@qq.com

S. Shen

e-mail: 359031346@qq.com

L. Qiu

e-mail: 313962503@qq.com

C. Zhang

e-mail: 1028122193@qq.com

M. Chen

e-mail: chenmeng1987.love@163.com cartilage is an important factor to the normal movement of the joint, but before the application of MRI technology in clinic, articular cartilage imaging is almost blind. Therefore, before the use of MRI, the thesis of imaging of articular cartilage and its diseases can be said to be very rare.

\section{Current Status in China}

In 1996, a review article [1] "MR Imaging Study Of Articular Cartilage" summarized early experience with MRI of articular cartilage by stating that "although the role of MRI in the evaluation of articular cartilage remains undefined, the ideal sequences may be fat suppression or MTC 3D-GRE". So far, about 116 articles concerning cartilage image study had been published on the nucleus journals in China since 2000. And research in this field is steadily increasing since 2003 (Fig. 1). Statistically, there are 73 clinical application articles, 31 experimental articles, and 12 reviews.

Cartilage pathology may be the result of degeneration or injury. Many imaging methods are available to assess cartilage, and MRI of cartilage articles accounts for $89.7 \%$, while ultrasound only accounts for $9.5 \%$. There is no doubt that MRI is the most important method for cartilage evaluation at present. Conventional radiography can be used to detect gross loss of cartilage, evident as narrowing of the distance between the two adjacent bones of a joint, but it does not show cartilage directly. CT provides information limited to the contour of the cartilage surface, while MRI, with its excellent soft-tissue contrast, is the best imaging technique currently available for the assessment of articular cartilage [2]. 
Fig. 1 Published articles on cartilage imaging in China

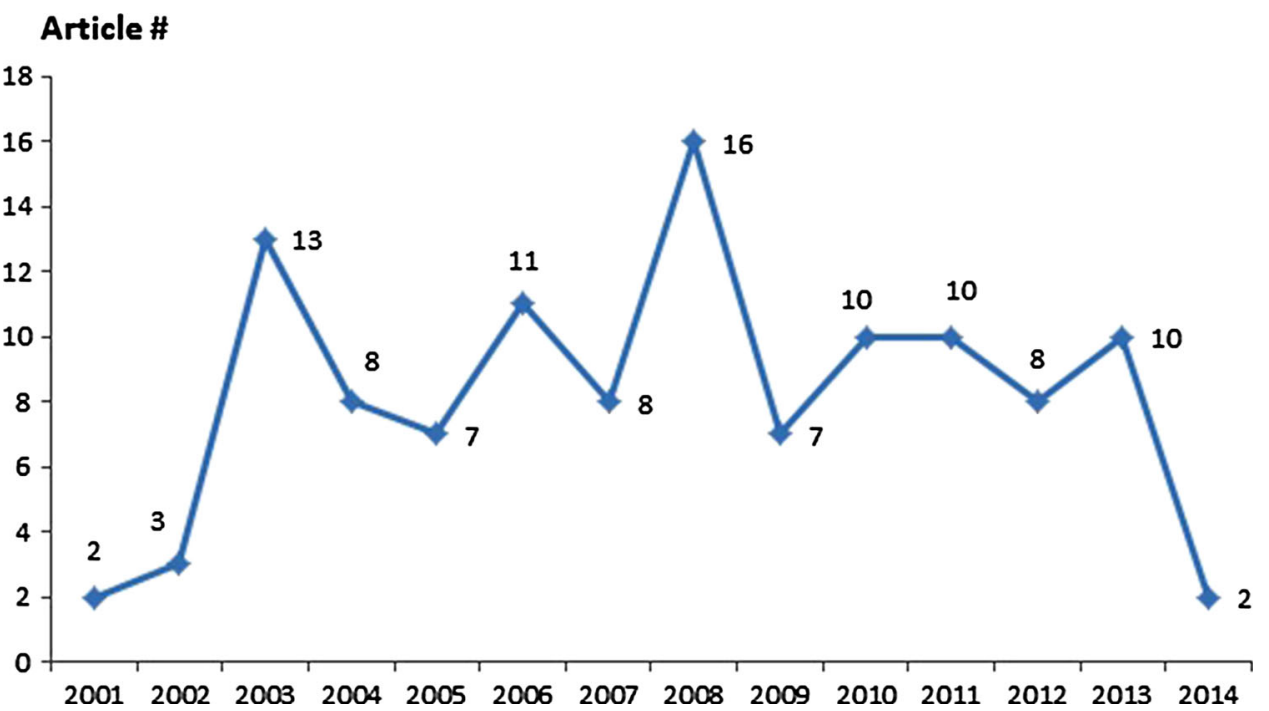

In recent years, articles published in the journal of China on cartilage mainly involve three aspects: (1) the MR sequences of cartilage; (2) MRI manifestations and diagnosis of cartilage disease; and (3) using MRI to reveal the biochemical composition, histological characteristics, and biomechanical structure of cartilage. A large number of researches that are invested in the development of these morphologic and quantitative imaging techniques are motivated by new therapeutic modalities, such as cartilage repair and pharmacological therapy.

\section{Conventional MRI Methods}

\section{D Fast Spin-Echo Imaging}

$\mathrm{T}_{1} \mathrm{WI}, \mathrm{T}_{2} \mathrm{WI}$, PDWI with or without suppression (There were 50 articles on this sequence in recent years).

Yin Jingjing et al. [3] found that sagittal fat saturation $T_{1}$ sequence can depict the knee meniscus and articular cartilage better than other scan sequences, but it provided poor contrast between cartilage and joint effusion, while $\mathrm{T}_{2} \mathrm{WI}$ provided better contrast than $\mathrm{T}_{1}$. FS- $\mathrm{T}_{2} \mathrm{WI}$ could be used to detect cartilage defects; FSE $\mathrm{PD} / \mathrm{T}_{2} \mathrm{WI}$ sequences provide excellent SNR and CNR of cartilage defects [4]. In osteoarthritis, these conventional sequences were suited for morphologic assessments and of articular cartilage and other structures around it [5-7].

\section{D Gradient-Echo Techniques}

Three-dimensional spoiled gradient-recalled echo imaging with fat suppression (3D-FS-SPGR) (There were 25 articles on this sequence in recent years).
Dual-echo steady state (DESS) (There were 3 articles on this sequence in recent years).

\section{$3 D-F S-S P G R$}

Plenty of research showed that 3D-FS-SPGR is the standard sequence for morphologic imaging of cartilage, and it offers higher SNR and CNR than 2D techniques $[8,9]$ (Fig. 2); meanwhile, it is useful for cartilage volume and thickness measurement [10-16]. But, it also has disadvantages; it does not adequately highlight surface defects with fluid and does not allow thorough evaluation of other joint structures such as ligaments or menisci [17].

\section{DESS}

Studies have demonstrated that the usefulness of 3D DESS imaging for the morphologic assessment of cartilage provided higher SNR and CNR compared with other sequences [18] (Fig. 3). The acquisition time for DESS is shorter than that for 3D-FS-SPGR, besides, it allowed quantitative assessment of cartilage thickness and volume with good accuracy [19].

\section{New MRI Methods}

$\mathrm{T}_{2}$ Relaxation Time Mapping (There were 16 articles on this sequence in recent years)

The $T_{2}$ relaxation time of cartilage is sensitive to its matrix [20]. It reflects the interactions among water molecules and surrounding macromolecules. It may reveal the areas of increased or decreased water content that correlate with cartilage damage [21]. It also can indicate component abnormality of articular cartilage without morphology 


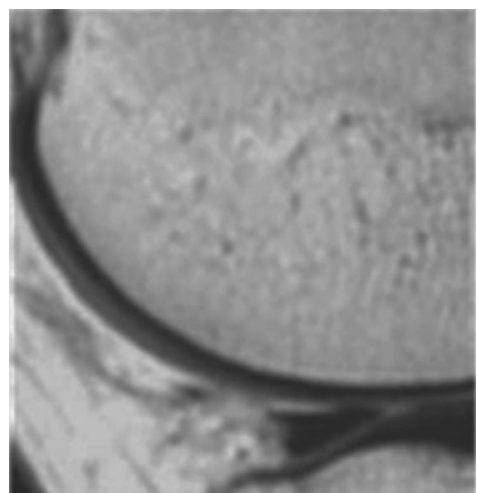

TIWI
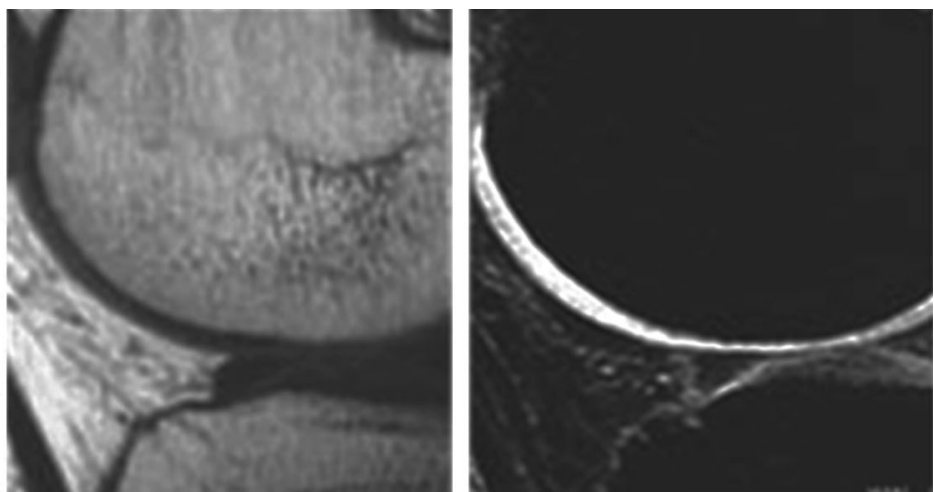

T2WI

3D-FS-SPGR

Fig. 2 Human cartilage of knee

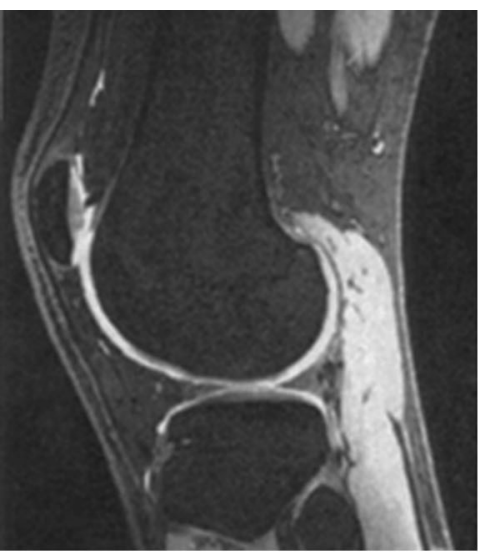

DESS

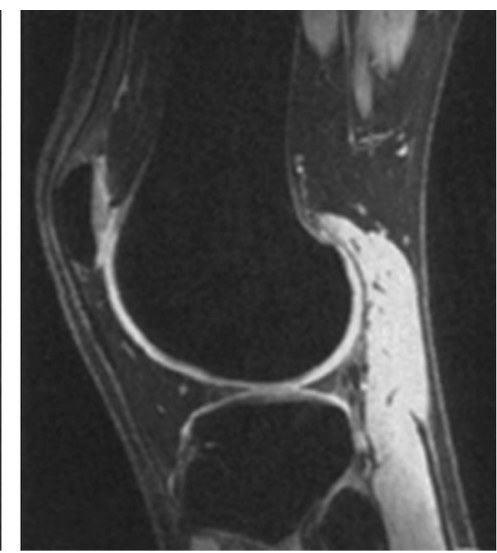

MEDIC

\section{TrueFISP}

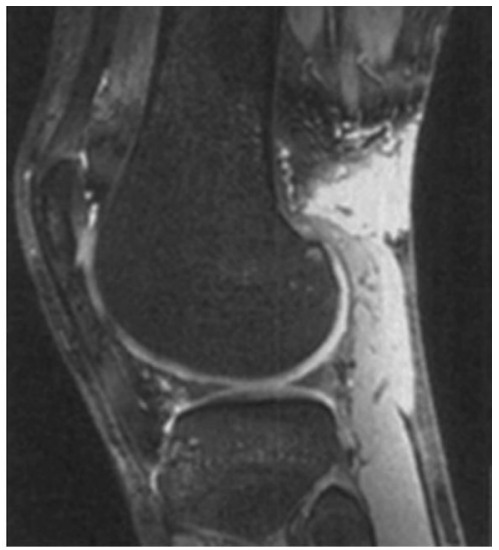

Fig. 3 The lateral condyle of knee joint [18] (Color figure online)

change of early OA. Chen Qichun et al. found that " $T_{2}$ mapping imaging (Fig. 4) of articular cartilage can monitor early cartilage degeneration prior to morphologic changes, which has a high value to diagnose and detect early cartilage injury in the early stage of OA. The $\mathrm{T}_{2}$ value of cartilage is influenced by the weight-bearing condition, and the $T_{2}$ value of weight-bearing site is higher than that of non-weightbearing site" [22•]. The $\mathrm{T}_{2}$ value of the articular cartilage could increase significantly with cartilage deterioration, which was highly related to the degree of cartilage degeneration and could be considered as a sensitive method to detect early cartilage degeneration [23-27].

DWI (There were 2 articles on this sequence in recent years)

Diffusion-weighted imaging is based on the motion of water molecules. It can detect water molecular diffusion state of tissue (degrees of freedom and direction). The diffusion of water in cartilage reflects tissue's biochemical structure and architecture. The apparent diffusion coefficient (ADC) is low in healthy cartilage because the diffusion of water molecules is restricted by cartilage matrix. However, disruption of the cartilage matrix results in an enhanced water mobility, which increases the ADC of cartilage. This research also confirmed that the ADC av of the healthy people was lower than that of OA group (Fig. 5). DWI is a useful method in detecting early cartilage injury which cannot be shown on routine sequences [28]. ADC value of patellar cartilage in early CMP was obviously increased, and DWI had a high clinic value in diagnosis and follow-up of CMP [29].

Delayed Gadolinium-Enhanced MRI of Cartilage (dGEMRIC) (There were 8 articles on this sequence in recent years)

After intravenous injection of Gd-DTPA, it penetrates cartilage and concentrates where the cartilage 
Fig. $4 T_{2}$ mapping colored map of the lateral condyle cartilage [22] a, b female, 35, normal volunteer; the cartilage signal was uniform, showed light green in color; c, d male, 52, early OA patient; the cartilage signal was inhomogeneous, showed yellow or reddish yellow in color
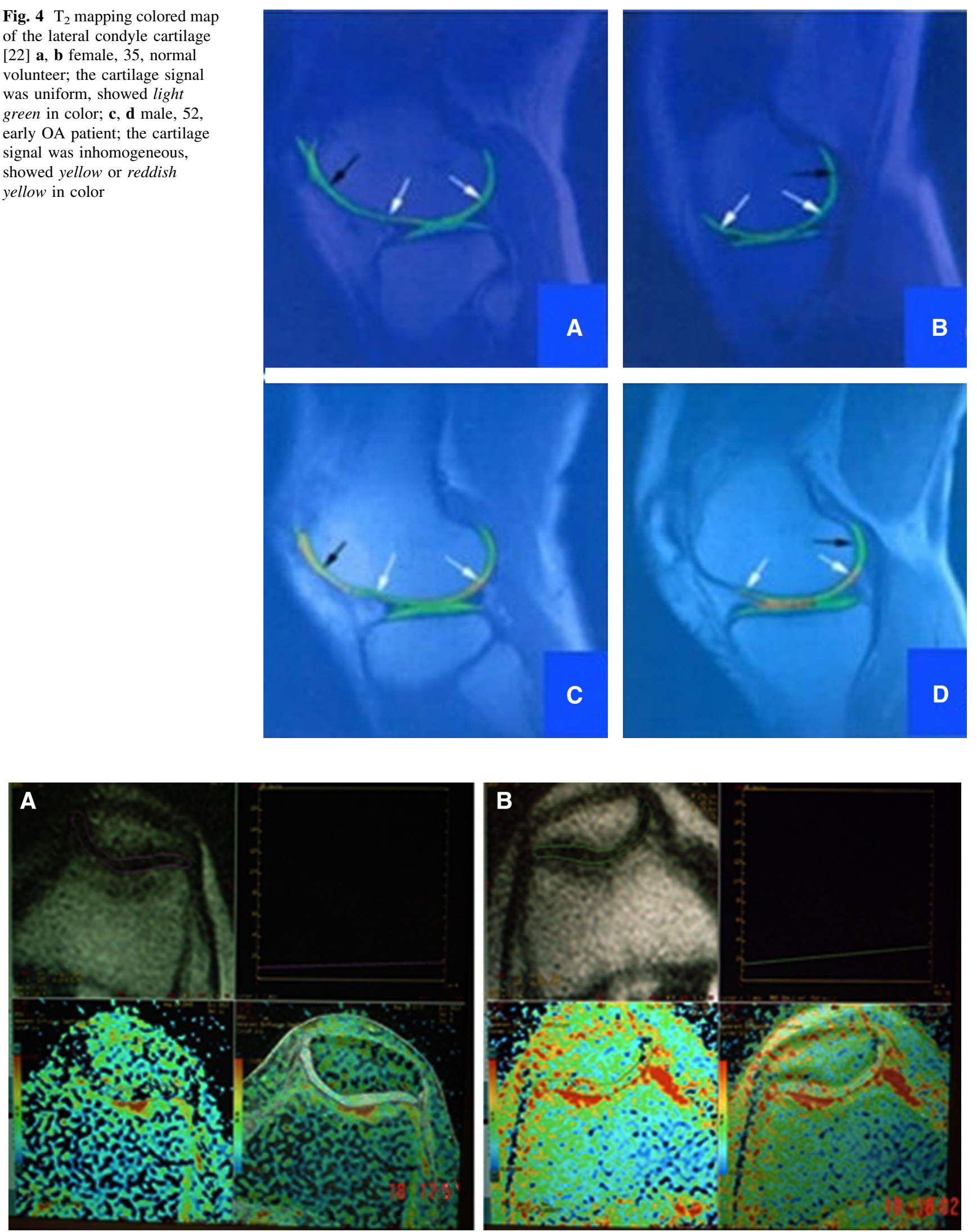

Fig. 5 ADC map of patella cartilage. a. healthy people: ADC av: $(4.36 \pm 6.09) \times 10^{-4} \mathrm{~mm}^{2} / \mathrm{s}$; b. OA people: ADC av: $(13.7 \pm 6.21) \times$ $10^{-4} \mathrm{~mm}^{2} / \mathrm{s}$ 


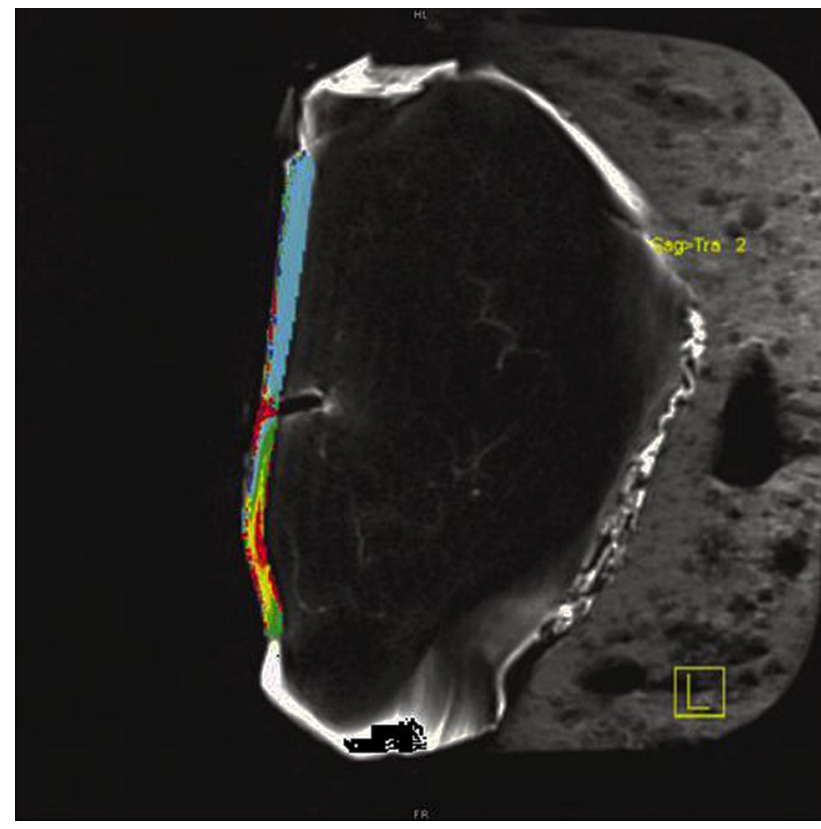

Fig. 6 Bovine cartilage (dGEMRIC). The upper cartilage was treated by trypsin, and the second half was treated as control [52・•]

glycosaminoglycan content is relatively low. Subsequent T1 imaging, which is reflective of Gd-DTPA concentration, therefore yields an image depicting glycosaminoglycan distribution. This technique is referred as delayed gadolinium-enhanced MRI of cartilage (dGEMRIC) [30-32] (Fig. 6).

An animal research had revealed that "SIR on early and delayed Gadolinium-enhanced MRI has the ability in evaluating the early stage change of cartilage degeneration" [33]. Doses of Gd-DTPA had important effect on cartilage $\mathrm{T}_{1}$ value, contrast ability, and delay time of dGEMRIC [31]. In 2009, a clinical study indicated that "dGEMRIC can be used to diagnose whether the biochemical ingredients of cartilage were lost by means of quantitating the GAG. It has a potential feasibility to diagnose the early stage of cartilage degeneration" [34].

$\mathrm{T}_{1} \rho$ Mapping (There were 5 articles on this sequence in recent years)

$\mathrm{T}_{1} \rho$ relaxation time is similar to $\mathrm{T}_{2}$ relaxation time. The interactions between motion-restricted water molecules and their local macromolecular environment can be monitored by measuring $T_{1} \rho$ value, making this a promising technique for assessing the composition of cartilage, since its extracellular matrix provides a motion-restricted environment for the water molecules. Thus, the changes to the extracellular matrix, such as proteoglycan depletion, may alter $\mathrm{T}_{1} \rho$ values measured in cartilage [35]. In the damaged hyaline cartilage, it demonstrates higher $T_{1} \rho$ value than normal cartilage, thus, $\mathrm{T}_{1} \rho$ measurement could be used as a noninvasive early evaluation method for cartilage disease [36].

\section{Clinical Application}

The most important clinical applications for MRI are the assessment of cartilage in osteoarthritis, chronic or acute osteochondral injury, osteochondritis, chondromalacia patellae, and inflammatory arthropathies. In addition, cartilage imaging is required to monitor the treatment's effect after invasive cartilage repair procedures or conservative therapies (including pharmacological therapies).

\section{Degenerative Disease}

Osteoarthritis is an important cause of disability in China and is marked by degeneration of articular cartilage. Early degenerative lesion may be seen on conventional MRI [37]. It appears to be the changes of cartilage contour (such as fibrosis and irregular surface) and cartilage thickness (including thinning or thickening) [38]. While, advanced degenerative lesion shows multifocal thinning or missing of the cartilage [39]. By comparing articular cartilage thickness between OA cases and healthy volunteers, the articular cartilage thickness in a bulk of cartilage weighloading surface attenuated gradually, following with the aggregated OA disease [40].

Cartilage lesions on MRI are often graded on Noyes scale: 0 Normal; 1 Signal change (increased T2); 2 Partialthickness defect $<50 \% ; 3$ Partial-thickness defect $\geq 50 \%$; and 4 Full-thickness defect [41, 42].

Many MRI sequences are sensitive to the biochemical changes within cartilage [43, 44]. More specifically, $\mathrm{T}_{2}$ value was able to provide information about the change of cartilage collagen, and increased $T_{2}$ is most commonly associated with cartilage damage (collagen destruction); $\mathrm{T}_{1} \mathrm{Gd}$ was mainly correlated with the content of GAG, and the lower GAG leads to decreased $\mathrm{T}_{1}$ value [45•]. ADC value of patellar cartilage in early chondromalacia patellae is obviously increased [29].

\section{Traumatic Chondral Injuries}

Acute injury to cartilage can be characterized by MRI [46, 47]. Cartilage injury lesions on MRI showed partial or full cartilage fracture, avulsion or subchondral bone marrow contusion, and edema or subchondral fracture [48, 49]. Discovery signal changes of subchondral bone marrow 
should be alert to the corresponding articular cartilage injury or disease [50]. The isolated traumatic cartilage fracture does not exist in fact. And usually, the traumatic injury of articular cartilage should be combined with the subchondral bone contusion [51]. The assessment of MRI and arthroscopy in acute articular cartilage injury is consistent. Combined with arthroscopy, MRI can succeed in assessing the extension and degree of acute articular injury and allowing treatment planning [52••, 53]. An experimental research revealed that the increase of MMP13 expression of the covering cartilage of bone flap was of significance for clinical therapy [54].

\section{Inflammatory Arthropathies}

MRI can show the pathological changes of RA in early stages, such as chondral damage [55]. A research on animal

Our team's achievements:

2002-2003: Compared the advantage or shortage of several scan sequences in MRI of articular cartilage and determined the optimal series. From this we concluded that 3D-FS-SPGR could show the cartilage clearly than other sequences, the value of cartilage thickness with 3D-FS-SPGR was more accurate.

2003: Evaluated the diagnosis and differential diagnosis among chronic cartilage lesions on the plain films and MRI. A combination of the plain films and MRI was the best method for examining the joints and cartilages. 3D-FS-SPGR was the best sequence for the diagnosis of cartilage lesion.

2006: Evaluated the clinical significance of quantitative measurement ADC value of patella cartilage on MR diffusion weighted imaging (DWI). The ADC value of healthy people was lower than of the OA group, DWI was a useful method in detecting early cartilage injury which cannot be showed on routine sequences.

2007: 3D-FS-SPGR sequence is more sensitive than SE-Tl-WI sequence in detecting the change of cartilage signal intensity. SIR on early and delayed Gadolinium-enhanced MRI has the ability in evaluating the early stage changing of cartilage degeneration.

2008: T2* map could give a quantitative criteria for abnormal microscopic content of water and the structure change of articular cartilage . T2* map is a useful method in detecting the cartilage injury.<smiles>C=[Te]</smiles>

2013:An animal study reveals that $T_{2}$ and $T_{1}$ mapping may be useful in the early diagnosis of cartilage degeneration. The changes in collagen content of the cartilage was related to $T_{2}$ relaxation time. Whereas $T_{1}$ relaxation time was affected by both collagen and proteoglycan.

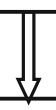

2014:An animal research and clinical study reveals that T1 $\rho$ imaging technique has the better feasibility and sensitivity to the early diagnosis of articular cartilage degeneration, it might perform the prospective and quantitative evaluation to the prophase degenerative cartilage and the injuries should happen in further. 
models of pyogenic arthritis induced by intra-articular injections of Staphylococcus aureus suspension revealed that diffuse signal increased on FSE $\mathrm{T}_{2} \mathrm{WI}$ was the earliest sign of articular cartilage involvement in pyogenic arthritis. The changes of both signal intensity and morphology of articular cartilage can reflect histopathologic abnormality [56]. In addition, inflammatory infiltration in subchondral bone is the early sign of adjacent cartilage injury [57, 58].

\section{Cartilage Repair Procedures}

With the development of arthroscopic surgery and cartilage transplantation, all kinds of repair technologies about articular cartilage defect are getting matured. We should not only find the accurate location of articular cartilage abnormalities but also the MRI evaluation of operation effect. MRI also played an essential role in the assessment of cartilage volume [59]. Deng Xia et al. [60] got a conclusion that MRI provided a noninvasive way for monitoring the process of repair after AOT.

Whether the result is from degeneration or injury and inflammation, MRI offers a noninvasive means of assessing the degree of damage to cartilage and adjacent bone and of measuring the effectiveness of treatment.

\section{Future Directions}

(1) Technologic advances in high-field imaging, pulse sequence development, and the application of MRI contrast agents.

(2) To observe cartilage transplantation and drug therapeutic effect using MRI.

(3) Molecular imaging: to observe cartilage structural and biochemical changes.

\section{Compliance with Ethics Guidelines}

Conflict of Interest Dr. Fei Wang, Dr. Si Shen, Dr. Lin Qiu, Dr. Cici Zhang, Dr. Meng Chen, and Dr. Sirun Liu each declare no potential conflicts of interest.

Human and Animal Rights and Informed Consent This article does not contain any studies with human or animal subjects performed by any of the authors.

\section{References}

Recently published papers of particular interest have been highlighted as:

- Of importance

•- Of major importance
1. Zhou G. MR imaging study of articular cartilage. Foreign Med Sci Clin Radiol Fascicle. 1996;2:90-3.

2. Han C, Sun Y, Jian L, et al. Best pulse sequence of MRI in demonstrating the articular cartilage layering of normal knee joint. Radiol Pract. 2007;22(7):747-9.

3. Yin J, Li W, Yang Z, et al. Value of sagittal SE T1 fat saturation scan sequences in evaluating knee meniscus and articular cartilage. Chin J Med Imaging Technol. 2003;19(9):1225-7.

4. Li S, He C, Wu Z, et al. Study of MR sequence in detecting hyaline cartilage defects of the knee joint. Chin J Radiol. 2003;37(11):967-72.

5. He C, Li S, Sun W, et al. MR imaging sequences in hyaline cartilage defects: an experimental study. J Clin Radiol. 2003;22(1):67-72.

6. He C, Li S, Bai X, et al. Study of comparing the MR sequence in detecting hyaline cartilage defects: comparing with arthroscopy. Pract Radiol. 2003;19(1):53-6.

7. Liu S, Zhu T, Huang L, et al. Imaging diagnosis of the articular cartilage disorders. Chin J Radiol. 2003;37(11):973-8.

8. Leng X, Liu S, Chen D, et al. Technique of MRI in imaging of articular cartilage in the knee. China JMIT. 2002;18(9):933-5.

9. Lin Z, Zhai L, Chen Y, et al. Comparative study of MR sequences on articular cartilage in the knee. Chin Clin Med Imaging. 2011;22(10):747-50.

10. Sun W, Li C, He C, et al. Clinical study of MR imaging sequence in hyaline cartilage. Chin J Med Imaging Techol. 2003;19(8): 1021-3.

11. Liu H, Sun Y, Du X, et al. Different MR sequences in evaluation of abnormalities of patellofemoral joint cartilage. Chin J Med Imaging Technol. 2002;18(5):471-3.

12. Sun Y, Du X, Zhou D, et al. Evaluation of abnormalities of patellofemoral joint cartilage by 3D-FS-FSPGR echo sequences. China J MIT. 2002;18(9):930-2.

13. Luo X, Hua L, Ding A, et al. Value of 3D-FS-SPGR sequence associated with $3 \mathrm{D}$ reconstruction in cartilage defects of knee. Chin Clin Med Imaging. 2005;16(3):153-5.

14. Yue Y, Han H. Application of improved FS-3D-TrueFISP sequence in diagnosis of hyaline cartilage defects in knee joint. Chin J Med Imaging Technol. 2007;23(1):105-8.

15. Gu F, Zhang X. The study of selective water excitation in the MR imaging of articular. Chin J Radiol. 2007;41(12):1299-303.

16. Leng X, Liu S, Huang L, et al. Normal articular cartilage of the adult knee: evaluated with multiple MR sequences imaging. J Pract Radiol. 2003;19(1):57-60.

17. Ai F, Li X, Zhang W, et al. Comparison of 3D FIESTA and fat suppressed 3D SPGR MRI in evaluating knee cartilage lesions. J Clin Radiol. 2010;29(4):501-5.

18. Yang Q, Zheng Z. Comparison of MR DESS, MEDIC, True FISP sequences for knee cartilage. Chin J Med Imaging Technol. 2010;26(3):535-7.

19. Jiang S, Gong M, Lv Y, et al. Comparison of different 3T MR sequences in detecting articular cartilage. Chin Comput Med Imag. 2010;16(4):335-9.

20. Zhou Z, Shan H, Zou X, et al. Regional changes of T2 relaxation on porcine patellar cartilages in vitro by means of degradation enzymatically at 7.0T MR. Chin J Med Imaging Technol. 2009;25(3):355-8.

21. Yang H, Wang R, Li F, et al. Comparative study between MR T2-mapping of cartilage and severe classification of plain film in knee osteoarthritis. Radiol Pract. 2007;11:1158-60.

22. - Chen Q, Zou Y, Wang D, et al. The application of MRI T2 mapping imaging in the early osteoarthritis of the knee. J Clin Radiol, 2012,31(1): 81-85. T2 mapping imaging of articular cartilage can monitor early cartilage degeneration prior to 
morphologic changes, which has a high value to diagnose and detect early cartilage injury in early stage of $O A$.

23. Wang $\mathrm{H}$, Hao D, Xu W, et al. 3.0 T MRI T2-mapping evaluates knee cartilage degeneration. Chin J Magn Reson Imaging. 2012;3(4):245-9.

24. Zhou Z, Shan H, Zou X, et al. T2 mapping and quantitative assessment of articular cartilage at 7.0T MR. Chin J Imaging Technol. 2008;24(2):171-5.

25. Lu Z, Yao W, Hu S, et al. Application in assessment of osteoarthritis in rabbit knee's articular cartilage with MR T2 mapping. Chin Comput Med Imag. 2008;14(5):450-5.

26. Cao M, Zhang J. Evaluation on the relationship between meniscal degeneration and osteoarthritis with T2-mappig. Chin J Med Imaging Technol. 2012;28(6):1204-7.

27. Wu K, Wang $\mathrm{T}$, Liang $\mathrm{H}$, et al. $\mathrm{T} 2$ mapping of knee cartilage damage at 3.0 T MR imaging. Chin J Magn Reson Imaging. 2013;4(3):210-4.

28. Liu S, Zhu T, Chen $\mathrm{H}$, et al. MR diffusion weighted imaging study on patella cartilage applied to osteoarthritis of knee joint. Chin J Radiol. 2006;40(10):1098-101.

29. Yang H, Wang R, Li F, et al. Comparative study of MR T2mapping and DWI in early chondromalacia patellae. J Pract Radiol. 2008;24(3):336-42.

30. Li X, Qi J, Wang R, et al. The study of dynamic gadoliniumenhanced MR imaging in normal growth cartilage. J Clin Radiol. 2006;25(10):971-4.

31. Li Y, Li X, Wang R, et al. Dynamic and delayed gadoliniumenhanced MRI: early ischemia of the capital femoral epiphysis in piglets. Chin J Med Imaging Computer. 2006;12(3): 209-12.

32. Zheng Z, Li J, Li X. Full-joint coverage delayed gadoliniumenhanced MRI of cartilage : preliminary study in vitro. Chin J Med Imaging. 2010;18(1):14-8.

33. Shen S, Liu S, Zhu T, et al. An initial experimental study-the value of gadolinium-enhanced MRI and delay enhanced MRI in detecting articular cartilage degeneration of the rabbit knee. Chin J Radiol. 2007;41(12):1394-400.

34. Zheng Z, Li X, Shang Y, et al. Influence of Gd-DTPA doses on delayed Gadolinium-enhanced MRI of cartilage: an in vitro study. Chin J Med Imaging Technol. 2009;25(3):339-42.

35. Song L, Liang B, Zhong J, et al. Value of dGEMRIC in diagnosing early cartilage degeneration of knee joint. Chin J Med Imaging Technol. 2009;25(6):1078-80.

36. Duvvuri U, Charagundla SR, Kudchodkar SB, et al. Human knee: in vivo $\mathrm{T} 1$ (rho)-weighted $\mathrm{MR}$ imaging at $1.5 \mathrm{~T}$-preliminary experience. Radiology. 2001;220(3):822-6.

37. Lu Y, Ding X, He C, et al. Knee osteoarthritis: the correlation between cartilage magnetic resonance imaging and matrix components expression. J Clin Radiol. 2008;27(12):1712-5.

38. Zhang Q, Yuan M. MRI finding of pure cartilage injury of the knee joint. J Clin Radiol. 2003;22(8):689-91.

39. Zhou Z, Shang H, Li Z, et al. Porcine patellar cartilages in vitro by means of trypsin digestion: 7.0T MR T1 $\rho$ weighted image and quantitative analysis. J Clin Rehabil Tissue Eng Res. 2009;13(37):7221-5.

40. Lin Z, Zhai L, Chen Y, et al. Clinical application of 3D-FS-SPGR sequence on the disease of knee articular cartilage. Chin J Clin Anat. 2011;29(3):296-300.

41. Wang S, Sun J, Mao C, et al. MR imaging diagnosis of the degenerative osteoarthrosis of the knee joints. Radiol Pract. 2004;19(4):250-2.
42. Li W, Lu Y, Ding X, et al. MRI features and clinical relative factors of asymptomatic adult knee cartilage lesions. Chin J Med Imaging Technol. 2009;25(11):2088-91.

43. Qu N, Yao W, Lu Z, et al. Measurement of T2 relaxation time in patella cartilage in diagnosis of osteoarthritis. Chin $\mathrm{J}$ Med Technol. 2008;24(12):1992-4.

44. Lu Y, Ding X, He C, et al. Articular cartilage of the knee: laminar appearance in MR imaging with histological findings. Chin J Med Imaging Technol. 2006;22(11):1799-802.

45. - Shen F, Lu Y, Ding X, et al. Magnetic resonance imaging of cartilage in knee osteoarthritis. Chin Comput Med Imag, 2011,17(1):54-58. With a suitable evaluation system, cartilage magnetic resonance can be effectively used in clinical diagnosis of $O A$.

46. Zheng $\mathrm{H}$, Li X, Qi J, et al. The diagnostic value of MR T2 mapping on early articular cartilage injury of knee. J Clin Radiol. 2008;27(9):1247-50.

47. Wang X, Gu J, Liu Y, et al. MRI diagnosis of the immature knee joint cartilage. Chin Med Imaging. 2011;22(1):59-61.

48. Sun Y, Cui J, Zhang Y, et al. MRI finding of juvenile acute pure cartilage fracture of knee joint. Chin J Radiol. 2007;41(12):1304-8.

49. Hu H, Lian J, Liu Y, et al. Analysis the MRI imaging of cartilage fracture in the knee joint. MMI. 2013;22(1):1-4.

50. Liu Y, Huang H, Tu Q. Role of low field MR in diagnosis of articular cartilage injury of knee and its comparison with arthroscopy. J Radiol Pract. 2009;24(4):430-3.

51. Pan S, Li Z, Sun H, et al. China female judokas with knee injuries:3.0T MRI analysis. J Chin J Med Imaging Technol. 2008;24(6):804-7.

52. $\bullet$ Zheng Z, Li X, Liu Y. The study of correlation between MR relaxation time and biochemical composition of cartilage. Chin J Med Imaging, 2010,18(4):336-341. Quantitative MR relaxation times had the ability to reveal early biochemical changes within cartilage. More specifically, T2 was able to provide information about the change of cartilage collagen; T1Gd was mainly correlated with the content of GAG, whereas $T 1$ was affected by both collagen and GAG.

53. Ma J. MR diagnosis of articular cartilage injury in the knee: compared with arthroscopy. Med J Chin People's Health. 2007;11:954-5.

54. Feng S, Sun X, Wang B, et al. Influence of movement on outcome of osteochondral fracture in rabbits: MRI features and MMP-13 expressions. Radiol Pract. 2010;25(4):358-61.

55. Cui Y, Liu Z, Huang H. MRI features in different sequences and pathological changes on rheumatoid arthritis in rabbit model. Chin J Med Imaging Technol. 2009;25(11):1957-60.

56. Zhang J, Wu Z, Fan G, et al. The MRI study of articular cartilage of canine with pyogenic arthritis correlated with biochemical result. Chin J Med Imaging. 2006;02:133-7.

57. Zhang J, Wu Z, Fan G, et al. MRI evaluation of acute articular cartilage injury of knee. China J Radiol. 2003;37(11):979-83.

58. Zhang J, Wu Z, Fan G, et al. Experimental MRI study of articular cartilage in pyogenic arthritis: correlated with histopathology. Chin J Med Imaging Technol. 2005;21(7):991-3.

59. Ma C, Zhang L, Wei C, et al. Repeatability and accuracy of quantitative knee cartilage volume measurement using semiautomated software at 3.0T MR. Chin J Med Imaging Technol. 2010;26(4):760-3.

60. Deng X, Xu J, Lu Q, et al. 3.0T MRI of autologous osteochondral transplantation in a goat model. Chin J Med Imaging Technol. 2008;24(11):1675-7. 